\title{
Clinical performance of the Roche Elecsys SARS- CoV-2 Antigen fully automated electrochemiluminescence immunoassay
}

\section{Gian Luca Salvagno}

Section of Clinical Biochemistry, University of Verona, Verona, Italy

\section{Laura Pighi}

Section of Clinical Biochemistry, University of Verona, Verona, Italy

\section{Simone De Nitto}

Section of Clinical Biochemistry, University of Verona, Verona, Italy

Giuseppe Lippi ( $\nabla$ giuseppe.lippi@univr.it )

Section of Clinical Biochemistry, University of Verona, Verona, Italy

\section{Short Report}

Keywords: COVID-19, SARS-CoV-2, Laboratory Medicine, Diagnosis, Immunoassay

Posted Date: October 6th, 2021

DOl: https://doi.org/10.21203/rs.3.rs-957329/v1

License: (c) This work is licensed under a Creative Commons Attribution 4.0 International License.

Read Full License

Version of Record: A version of this preprint was published at Practical Laboratory Medicine on January 1st, 2022. See the published version at https://doi.org/10.1016/j.plabm.2022.e00265. 


\section{Clinical performance of the Roche Elecsys SARS-CoV-2 Antigen fully automated electrochemiluminescence immunoassay}

\section{Gian Luca Salvagno ${ }^{1,2}$, Laura Pighi ${ }^{1}$, Simone De Nitto ${ }^{1}$, Giuseppe Lippi ${ }^{1}$}

1. Section of Clinical Biochemistry, University of Verona, Verona, Italy

2. Service of Laboratory Medicine, Pederzoli Hospital, Peschiera del Garda, Italy

Short title: Evaluation of Roche Elecsys SARS-CoV-2 Antigen Test

Type of article: Short Communication

Word count: 1050 text +1 Table +1 Figure

Corresponding author:

Prof. Giuseppe Lippi

Section of Clinical Biochemistry

University Hospital of Verona

Piazzale L.A. Scuro, 10

37134 Verona - Italy

Tel. 0039-045-8122970

Fax. 0039-045-8124308

Email: giuseppe.lippi@univr.it 


\section{Abstract}

Objective: We assessed the clinical performance of novel Roche Elecsys SARS-CoV-2 Antigen fully automated electrochemiluminescence immunoassay (ECLIA).

Design and Methods: We tested 160 subjects, 110 (68.8\%) with positive molecular test for SARS-CoV-2 infection in nasopharyngeal samples with Altona Diagnostics RealStar SARS-CoV-2 RT-PCR Kit and Roche Elecsys SARS-CoV-2 Antigen. The local imprecision was validated by analyzing three nasopharyngeal samples with different antigen concentration $(1.84,9.51$ and 423.30 TCID50/mL) for 20 consecutive times (intra-assay imprecision) or for 10 consecutive working days (inter-assay imprecision).

Results: The local intra- and inter-assay imprecision of Elecsys SARS-CoV-2 Antigen ECLIA was found to be comprised between $2.0-2.0 \%$ and $5.8-7.6 \%$, yielding to a total imprecision of $6.2-7.8 \%$. Highly significant correlation was found between Elecsys SARS-CoV-2 Antigen ECLIA and cycle threshold (Ct) values of SARS-CoV-2 $S$ and $E$ genes (both $\mathrm{r}=-0.91 ; \mathrm{p}<0.001$ ). The area under the curve (AUC), sensitivity and specificity of Elecsys SARS-CoV-2 Antigen ECLIA were 0.83, 0.43 and 1.00 in all samples, 0.99, 0.87 and 0.99 in those with both $\mathrm{Ct}$ values $<30$, as well as $1.00,1.00$ and 0.89 in samples with both $\mathrm{Ct}$ values $<25$.

Conclusion: Roche Elecsys SARS-CoV-2 Antigen ECLIA may be a surrogate of molecular testing for identification of super-spreaders.

Keywords: COVID-19; SARS-CoV-2; Laboratory Medicine; Diagnosis; Immunoassay. 


\section{Introduction}

Although the detection of severe acute respiratory syndrome coronavirus 2 (SARS-CoV-2) RNA remains the gold standard in coronavirus disease 2019 (COVID19) diagnostics, widespread usage of molecular tests is plagued by bottlenecks such as relatively limited throughput and long turnaround time. The poor availability of reagents for performing molecular assays in many countries is another of such drawbacks. According to a recent worldwide survey disseminated by the American Association of Clinical Chemistry (AACC), the large majority of testing facilities (i.e., up to $80 \%$ ) are engaged in strenuous challenges to perform routine or urgent SARSCoV-2 diagnostic testing due to staff shortage and difficulties in reagents supply [1], thus leaving many laboratories with large backlogs of untested samples. Owing to the paramount volume of diagnostics tests still needed to contrast the ongoing COVID-19 pandemic, rapid antigen tests are increasingly proposed as feasible alternatives for highthroughput and fast diagnosis of SARS-CoV-2 infections, especially in subjects carrying high viral load $[2,3]$. Therefore, the purpose of this study was to assess the clinical performance of the novel Elecsys SARS-CoV-2 Antigen immunoassay.

\section{Materials and Methods}

The study population consisted of all consecutive subjects referred with suspected COVID-19 (either symptomatic or for being close contacts of SARS-CoV-2 positive subjects) to the Laboratory Medicine Service of Pederzoli Hospital (Peschiera del Garda, Verona, Italy), between August 16 and September 15, 2021. A nasopharyngeal swab (Virus swab UTM Copan, Brescia, Italy) was taken at admission and immediately conveyed to the laboratory. SARS-CoV-2 molecular testing was performed with Altona Diagnostics RealStar SARS-CoV-2 RT-PCR Kit (Altona Diagnostics GmbH, Hamburg, Germany), a real-time reverse transcription polymerase 
chain reaction (rRT-PCR) based on double amplification and detection of SARS-CoV-2 $E$ and $S$ genes. The assay was performed with Bio-Rad CFX96 ${ }^{\mathrm{TM}}$ Deep Well Dx RealTime PCR Detection System (Bio-Rad Laboratories, Hercules, CA, USA), whilst results were classified as positive when cycle threshold $(\mathrm{Ct})$ values of both SARS-CoV$2 S$ and $E$ genes were $<45$.

SARS-CoV-2 antigen testing was performed with the novel electrochemiluminescence immunoassay (ECLIA) Roche Elecsys SARS-CoV-2 Antigen on Roche Cobas 6000 (Roche Diagnostics GmbH, Mannheim, Germany). This technique is based on a one-step double antibody sandwich assay for detecting SARSCoV-2 nucleocapsid antigen in nasopharyngeal and oropharyngeal swabs. The test sample is reactive when the cut-off index (COI) is $\geq 1$. According to manufacturer's declaration the limit of detection is 22 Median Tissue Culture Infectious Dose (TCID50)/mL, whilst the imprecision ranges between 1.9-3.5\%.

The clinical performance of Elecsys SARS-CoV-2 Antigen ECLIA was compared with results of molecular testing by Spearman's correlation, receiver operating characteristic (ROC) curve analysis, and calculation of diagnostic sensitivity and specificity. The local imprecision of the assay has also been validated, by analyzing three nasopharyngeal samples with different antigen concentration $(1.84,9.51$ and 423.30 TCID50/mL) for 20 consecutive times (intra-assay imprecision) or for 10 consecutive working days (inter-assay imprecision). Final results of clinical measurements were expressed as median and interquartile range (IQR). The statistical analysis was carried out with Analyse-it software (Analyse-it Software Ltd, Leeds, UK). This study was part of routine clinical laboratory operations for SARS-CoV-2 screening and diagnosis at the local facility, so that patient informed consent and Ethical Committee approval were unnecessary. The study was conducted in accordance with the Declaration of Helsinki, under the terms of relevant local legislation. 


\section{Results}

The local intra- and inter-assay imprecision of Elecsys SARS-CoV-2 Antigen ECLIA was found to be comprised between $2.0-2.0 \%$ and $5.8-7.6 \%$, yielding to a total imprecision of $6.2-7.8 \%$, thus slightly higher than that reported by the manufacturer. The final study population consisted of 160 patients (median age 38 years, IQR 24-58 years; 69 women), 110 of whom (68.8\%) positive at molecular testing (i.e., Ct values of both SARS-CoV-2 $S$ and $E$ genes $<45)$. A highly significant correlation was observed between values of Elecsys SARS-CoV-2 Antigen ECLIA and measurable Ct values of SARS-CoV-2 $S$ and $E$ genes (both r=-0.91; 95\% CI, -0.94 to $-0.87 ; \mathrm{p}<0.001$ ). The diagnostic performance of Elecsys SARS-CoV-2 Antigen ECLIA is summarized in table 1, either cumulative or stratified for the $\mathrm{Ct}$ values obtained in nasopharyngeal samples. The overall area under the curve (AUC) for detecting SARS-CoV-2 positive samples was 0.83 , with 0.43 sensitivity and 1.00 specificity at manufacturer's recommended COI $\geq 1$ (Figure 1a). Such performance considerably increased in detecting samples with higher viral load (i.e., Ct values of both genes <30), displaying an AUC of 0.99 , with 0.87 sensitivity and 0.99 specificity at $\mathrm{COI} \geq 1$ (Figure $1 \mathrm{~b}$ ). In samples with even higher viral loads (i.e., Ct values of both genes <25), Elecsys SARSCoV-2 Antigen ECLIA exhibited further improved diagnostic performance, with 1.00 AUC, 1.00 sensitivity and 0.89 specificity (figure 1c).

\section{Discussion}

More than 100 years after the dramatic Spanish flu pandemic which caused nearly 50 million casualties in 1918/19 [4], humanities is now challenged by COVID19, a new life-threatening viral disease caused by the beta coronavirus SARS-CoV-2, which has already infected over 230 million people and has caused approximately 5 
million deaths according to the Johns Hopkins University of Medicine Coronavirus Resource Center [5]. Such a paramount number of infections and deaths is placing an unprecedented pressure on clinical laboratories all around the world, which now struggle to provide timely test results for early and accurate diagnoses of SARS-CoV-2 infections to all those who need them.

Recent evidence convincingly suggests that widespread testing, also encompassing efficient screening of high-risk contacts of SARS-CoV-2 positive cases and their household members, represents an essential step toward effective control of local COVID-19 outbreaks [6,7]. Therefore, the use of SARS-CoV-2 antigen immunoassays represent a valuable perspective for largely enhancing the current testing capacity, provided that the assay has been clinically validated before its introduction into routine practice [3].

The results of our investigation, which entailed the clinical assessment of the novel, fully automated Elecsys SARS-CoV-2 Antigen ECLIA, attests that this technique provides remarkable performance not only for detecting subjects with high SARS-CoV2 viral load (i.e., AUC of 1.00 for $\mathrm{Ct}$ values of both $S$ and $E$ genes <25), but also as a potentially useful surrogate test for molecular diagnostics, since its displayed acceptable accuracy also as population screening test (AUC of 0.83 on all samples, with 1.00 specificity). Based on its remarkably high specificity, a positive test results would hence not necessarily require to confirm sample positivity with rRT-PCR, whilst the excellent sensitivity for detecting nasopharyngeal samples with SARS-CoV-2 Ct values $<25$ will enable to efficiently and timely identify positive subjects with high viral load. Notably, Gniazdowski et al reported that over $97 \%$ of SARS-CoV-2 viral cultures are negative in nasopharyngeal samples displaying Altona $\mathrm{Ct}$ values <26.2 [7]. At a similar $\mathrm{Ct}$ threshold, Elecsys SARS-CoV-2 Antigen ECLIA displayed 1.00 AUC, 1.00 sensitivity and 0.89 specificity, which would make it a potentially valuable surrogate of molecular 
testing for mass SARS-CoV-2 screening and for specific identification of superspreaders [8].

\section{Ethical approval}

All procedures performed in studies involving human participants were in accordance with the 1964 Helsinki declaration and its later amendments.

\section{Disclosure of interest}

The authors declare that they have no competing interest.

\section{Funding}

None.

\section{Author contributions}

All authors were involved in designing study, analysing the data and writing the manuscript.

\section{References}

1. American Association of Clinical Chemistry. COVID-19 Survey Results. Available at: https://www.aacc.org/science-and-research/covid-19-resources/aacc-covid-19testing-survey/full-survey-results. Last accessed, October 1, 2021.

2. Mattiuzzi C, Henry BM, Lippi G. Making sense of rapid antigen testing in severe acute respiratory syndrome coronavirus 2 (SARS-CoV-2) diagnostics. Diagnosis (Berl). 2020 Nov 26:dx-2020-0131. doi: 10.1515/dx-2020-0131. Epub ahead of print. 
3. Bohn MK, Lippi G, Horvath AR, Erasmus R, Grimmler M, Gramegna M, et al. IFCC interim guidelines on rapid point-of-care antigen testing for SARS-CoV-2 detection in asymptomatic and symptomatic individuals. Clin Chem Lab Med. 2021. doi: $10.1515 / \mathrm{cclm}-2021.0455$.

4. Taubenberger JK, Morens DM. The 1918 Influenza Pandemic and Its Legacy. Cold Spring Harb Perspect Med 2020;10(10):a038695.

5. Johns Hopkins University of Medicine Coronavirus Resource Center. COVID-19 Dashboard. Available at: https://coronavirus.jhu.edu/map.html. Last accessed, October 1, 2021.

6. Chas J, Nadal M, Siguier M, Fajac A, Denis M, Morand-Joubert L, Pialoux G. Broad-based SARS-CoV-2 testing program for healthcare workers in a primary care hospital in France. Infect Dis Now 2021;51:556-559.

7. Gniazdowski V, Morris CP, Wohl S, Mehoke T, Ramakrishnan S, Thielen P, et al. Repeat COVID-19 Molecular Testing: Correlation of SARS-CoV-2 Culture with Molecular Assays and Cycle Thresholds. Clin Infect Dis. 2020 Oct 27:ciaa1616. doi: 10.1093/cid/ciaa1616. Epub ahead of print.

8. Goyal A, Reeves DB, Cardozo-Ojeda EF, Schiffer JT, Mayer BT. Viral load and contact heterogeneity predict SARS-CoV-2 transmission and super-spreading events. Elife 2021;10:e63537. 


\section{Legend to Figure 1.}

Receiver Operating Characteristics (ROC) curves of Roche Elecsys SARS-CoV-2 Antigen fully automated electrochemiluminescence immunoassay (ECLIA) against molecular testing in (a) all nasopharyngeal samples, (b) nasopharyngeal samples with cycle threshold $(\mathrm{Ct})$ values $<30$ and (c) nasopharyngeal samples with cycle threshold $(\mathrm{Ct})$ values $<25$. 
Table 1. Clinical performance of Roche Elecsys SARS-CoV-2 Antigen fully automated electrochemiluminescence immunoassay (ECLIA) stratified according to cycle threshold $(\mathrm{Ct})$ values.

\begin{tabular}{|l|c|c|c|c|}
\hline Ct values & AUC & Sensitivity & Specificity \\
\hline & & & \\
\hline All samples & $0.83(95 \% \mathrm{CI}, 0.77-0.89 ; \mathrm{p}<0.001$ & $0.43(95 \% \mathrm{CI}, 0.333-0.525)$ & $1.00(95 \% \mathrm{CI}, 0.93-1.00)$ \\
\hline & & & \\
\hline$<30$ & $0.99(95 \% \mathrm{CI}, 0.98-1.00 ; \mathrm{p}<0.0001)$ & $0.87(95 \% \mathrm{CI}, 0.75-0.945)$ & $0.99(95 \% \mathrm{CI}, 0.95-1.00)$ \\
\hline & & & \\
\hline$<25$ & $1.00(95 \% \mathrm{CI}, 0.99-1.00 ; \mathrm{p}<0.001)$ & $1.00(95 \% \mathrm{CI}, 0.89-1.00)$ & $0.89(95 \% \mathrm{CI}, 0.82-0.94)$ \\
\hline & & & \\
\hline
\end{tabular}

AUC, area under the curve; $C t$, cycle threshold. 